\title{
Flexible Frequency-Band Reallocation Networks Using Variable Oversampled Complex-Modulated Filter Banks
}

\author{
Håkan Johansson and Per Löwenborg \\ Electronics Systems, Department of Electrical Engineering, Linköping University, 58183 Linköping, Sweden
}

Received 22 December 2005; Revised 17 May 2006; Accepted 16 July 2006

Recommended by Soontorn Oraintara

A crucial issue in the next-generation satellite-based communication systems is the satellite on-board reallocation of information which requires digital flexible frequency-band reallocation (FBR) networks. This paper introduces a new class of flexible FBR networks based on variable oversampled complex-modulated filter banks (FBs). The new class can outperform the previously existing ones when all the aspects flexibility, low complexity and inherent parallelism, near-perfect frequency-band reallocation, and simplicity are considered simultaneously.

Copyright (c) 2007 H. Johansson and P. Löwenborg. This is an open access article distributed under the Creative Commons Attribution License, which permits unrestricted use, distribution, and reproduction in any medium, provided the original work is properly cited.

\section{INTRODUCTION}

The future society foresees globally interconnected digital communication systems offering multimedia services, information on demand, and delivery of information (data) at high data rates and low cost and with high performance. Terrestrial networks could in principle meet the requirements on communication capacity due to the practically unlimited bandwidth provided by fiber optic cables, but this capacity is rarely available today. A large investment is required to bridge the distance between the local exchange and the customer. It is therefore internationally recognized that satellite systems will play an important complementary role in providing the global coverage required for both fixed and mobile communications [1-3]. However, to meet the requirements of the communication systems of tomorrow, it is imperative to develop a new generation of satellite systems, payload architectures, ground technologies, and techniques combining flexibility with cost efficiency. It is envisaged that the improvements required as to the capacity as well as complexity fall in the range of one and two orders of magnitude [1].

The European Space Agency (ESA) outlines three major standard architectures for future broadband systems [1]. Two of these are the distributed access network and professional user network which are to provide high-capacity point-topoint and multicast services for ubiquitous Internet access.
The satellites are to communicate with user units via multiple spot beams. In order to use the limited available frequency spectrum efficiently, the satellite on-board signal processing must support frequency-band reusage among the beams and also flexibility in bandwidth and transmitted power allocated to each user. Further, dynamic frequency allocation is desired for covering different service types requiring different data rates and bandwidths. An important issue in the next-generation satellite-based communication system is therefore the on-board reallocation of information. In technical terms, this calls for digital multi-input multi-output (MIMO) flexible frequency-band reallocation (FBR) networks (Frequency-band reallocation is also referred to as frequency multiplexing and demultiplexing.) which thus are critical components. Figure 1 illustrates the principle of FBR.

The following main requirements on the next-generation flexible FBR networks are identified.

\section{Flexibility}

Frequency bands of different and variable bandwidths must be handled.

\section{Low complexity and inherent parallelism}

The implementation complexity must be low. Further, the network (algorithm) itself should not impose restrictions 
Input data rate $1 / T_{\text {in }}$ samples/second $\quad$ Output data rate $1 / T_{\text {out }}$ samples/second

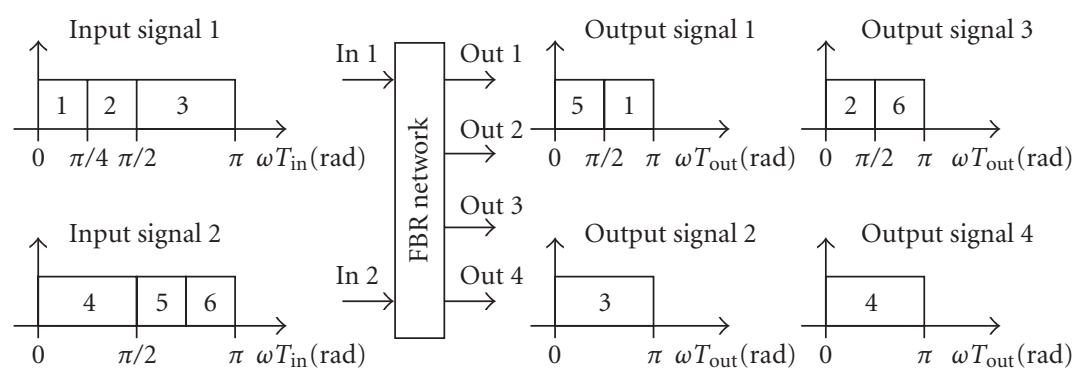

FIGURE 1: Illustration of frequency-band reallocation in the case of two input signals, four output signals, and six users. In practice, one must also include frequency guard bands between the subbands in order to make the network realizable (see Section 2).

on the feasible throughput. The implementation technology available should be the limiting factor. Meeting these requirements, high-throughput/low-power implementations can be obtained.

\section{Near-perfect frequency-band reallocation}

Near-perfect FBR means that each subband can be shifted to the new positions with small errors. By using an FBR network that is able to approximate perfect FBR as close as desired, the degradation of the overall system performance [typically measured in terms of bit-error-rate (BER)] due to these networks can be made as small as desired.

\section{Simplicity}

Simplicity means that the FBR network should be easily analyzed, designed, and implemented. Although this may not be strictly needed in order to arrive at a high-performance processor, it is naturally advantageous to keep everything as simple as possible.

\subsection{Contribution of the paper and relation to previous work}

The contribution of this paper is the introduction of a new class of flexible FBR networks based on variable oversampled complex-modulated filter banks (FBs). Compared to the existing FBR networks [4-6], the proposed ones can (1) outperform the regular complex-modulated DFT FBbased networks in terms of flexibility since that technique is totally inflexible, (2) outperform the tree-structured FBbased networks in terms of flexibility and complexity because tree-structured FBs in our environment only offer partial flexibility (although the title of [6] indicates full flexibility) and require a substantially higher complexity than that of modulated FBs (because most of the filtering does not take place at the lowest sampling rate involved), and (3) outperform the overlap/save DFT/IDFT-based networks $[4,5]$ in terms of near-perfect FBR since it is not known how to achieve this with that technique. Further, both treestructured FBs and overlap/save DFT/IDFT networks appear more complicated to analyze and design. In summary, the new technique can outperform the previously existing techniques when all the aspects flexibility, low complexity and inherent parallelism, near-perfect FBR, and simplicity are considered simultaneously. Thus, the technique presented here has the potential to become a standard solution for the next-generation satellite-based communications systems. It is noted that, although the proposed technique primarily targets a problem present in satellite-based communication, as outlined in [1], it is a general technique that can be used in any communication environment that requires transparent (bentpipe) flexible reallocation of information.

It is also noted that FBs have been used before in related contexts for partial reconstruction of spectra [7, 8], which is one of the functions of FBR networks, but neither of those papers addresses the general problem formulation of flexible FBR networks that is addressed in this paper. We also wish to point out that complex modulated filter banks have been studied in many papers before (see, e.g., [9-12]) but, again, neither of those papers addresses the problem dealt with in this paper. Finally, it is noted that parts of the material in this paper have been presented at a conference [13].

\subsection{Paper outline}

Following this introduction, Sections 2-5 are devoted to the proposed single-input single-output (SISO) networks whereas Section 6 points out the necessary modifications for obtaining the proposed MIMO networks. The reason why the main part of the paper considers the SISO case, despite the fact that a practical multicast system requires a MIMO network, is that it is beneficial to first understand and solve the SISO network case. This is because the SISO network case is simpler and a properly designed FBR SISO network can be utilized in MIMO networks. In this way, the analysis and synthesis of MIMO networks are greatly simplified.

\section{FLEXIBLE FBR SISO NETWORK}

The section begins with the problem formulation and then introduces the proposed flexible FBR network. 
Q-number of granularity bands

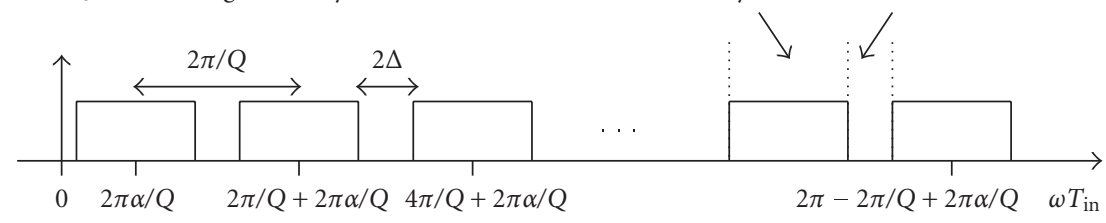

(a)

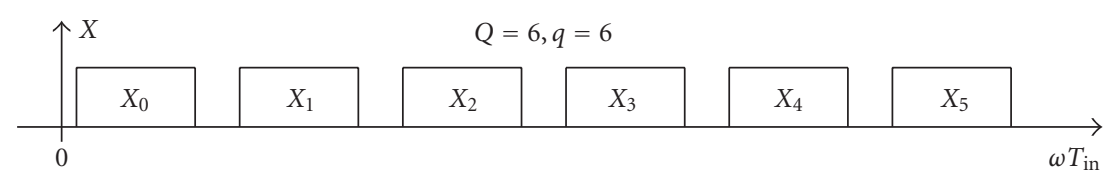

(b)

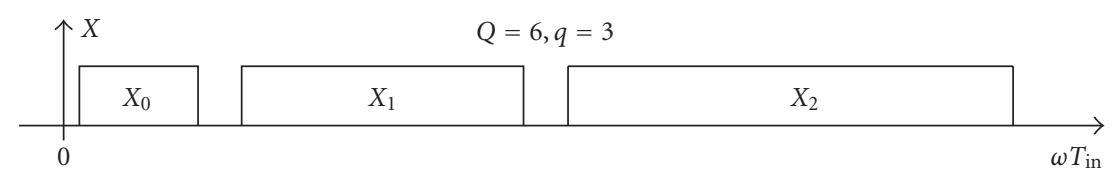

(c)

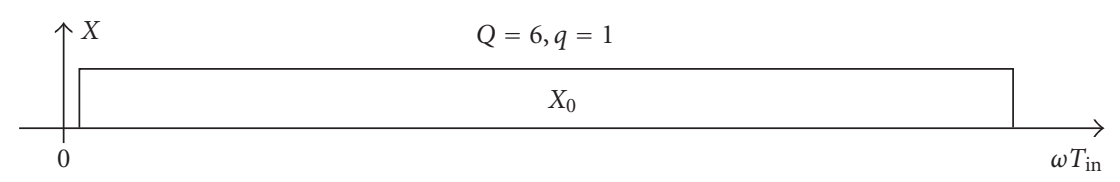

(d)

FIGURE 2: Granularity bands and typical input signals.

\subsection{Problem formulation}

The problem addressed here was outlined in [1] and is based on multiple frequency time division multiple access (MF/TDMA) schemes. The input signal is divided into $Q$ fixed granularity bands. Any user can occupy one or several of these granularity bands. The input signal thus contains an on-line variable (adjustable) number of user subbands $q$, where $1 \leq q \leq Q$. In the extreme cases, $q=Q$ and $q=1$, as illustrated in Figures 2(b) and 2(d), respectively, for $Q=6$. The case with $q=3$ and $Q=6$ is illustrated in Figure 2(c). It is stressed that $q$ is an on-line variable and its value can thus be changed during operation by an external controller. In addition, we assume full flexibility which means that all possible subband decompositions and reallocation schemes can occur. Furthermore, guard bands (transition bands) in frequency are assumed in order to ensure the network to be realizable in practice. This is also depicted in Figure 2. Guard bands are only present between different user subbands, not within a user subband.

The function of the flexible FBR SISO network is thus three-fold: it should (1) separate the input signal into the user subbands, (2) shift the user subbands in frequency to the desired positions, and (3) combine the frequencyshifted user subbands into the output signal. In principle, this function can be implemented through a bank of on-line adjustable-bandwidth filters for the signal separation, and time-varying complex-valued multiplications (modulators) for the frequency shifts. A straightforward implementation of the adjustable-bandwidth filters and time-varying multiplications would however result in a very high implementation cost. To solve the problem in a much more efficient way, we propose a new flexible FBR network based on oversampled complex-modulated FBs.

\subsection{Proposed network}

We introduce the flexible FBR SISO network shown in Figure 3. This scheme makes use of an $N$-channel analysis filter bank with fixed analysis filters $H_{k}(z)$ for splitting the input signal into $N$ subbands, and downsampling and upsampling by $M$ together with an $N$-channel synthesis filter bank with adjustable synthesis filters $G_{k}(z)$ for generating frequency shifts (i.e., redirecting the subbands to the desired output positions) as well as recombination of FB subbands into the $q$ shifted user subbands $y_{r}(n), r=0,1, \ldots, q-1$. In the SISO case, all $y_{r}(n)$ are finally summed to produce the single output $y(n)$, but in the general MIMO case, different $y_{r}(n)$ can be directed to different outputs (see Section 5).

The scheme in Figure 3 is the basis and it is used for analysis and design purposes. However, because the synthesis filters $G_{k}(z)$ are adjustable, they are not used in the final implementation because implementation of such filters becomes quite expensive. Therefore, a main point of this paper is to show that, with appropriate choices of filters and parameters in the FBR network, it is possible to implement the same 


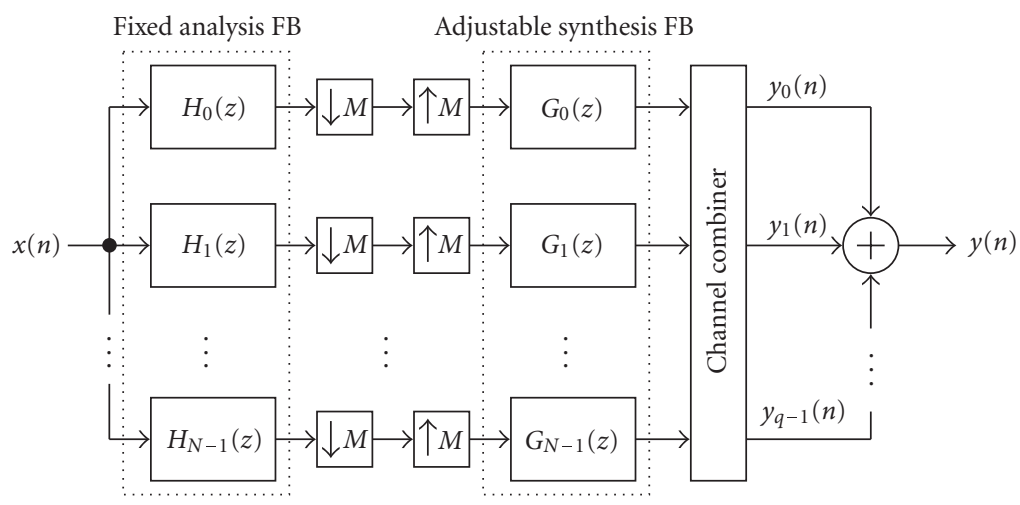

Figure 3: Proposed flexible FBR SISO network. The adjustable synthesis FB can be efficiently implemented using a fixed FB and a variable channel switch as indicated in Figure 4.

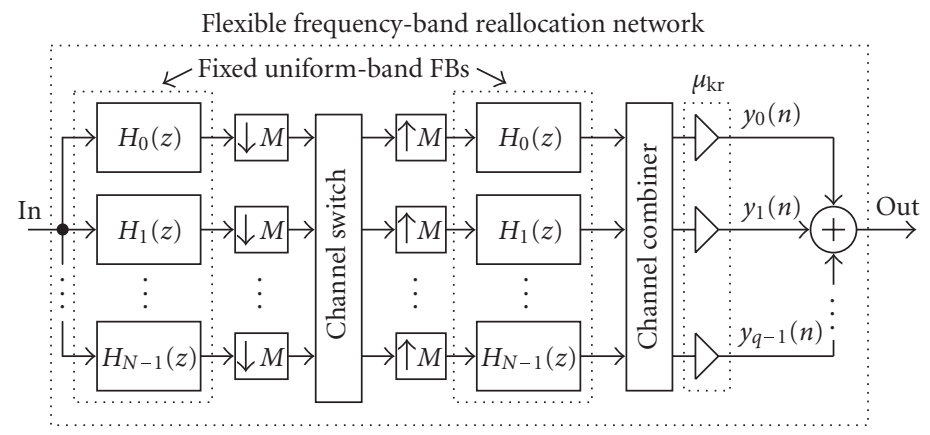

FIGURE 4: Efficient implementation of the proposed flexible FBR SISO network in Figure 3 using a fixed FB and a variable channel switch. With an appropriately chosen prototype filter order, all $\mu_{k r}$ become equal to unity.

function using instead a variable channel switch and fixed FBs according to the proposed scheme in Figure 4 where the output from the analysis filter $H_{k}(z)$ is connected to the input of the synthesis filter $H_{c_{k r}}(z)$, with $c_{k r}$ being given by (16) and $\mu_{k r}$ being adjustable phase rotations given by (17) in Section 2.5. In this way, the complexity can be reduced substantially, as fixed filters are considerably less complex to implement in hardware compared to adjustable filters. Furthermore, the fixed analysis (synthesis) FB can be implemented using only one filter block and an IDFT (DFT) block, and all $\mu_{k r}$ become unity for an appropriately chosen filter order. In all, this results in a very efficient realization with retained full flexibility. The key to this efficient solution is to make use of oversampling to avoid channel aliasing, more channels than granularity bands, and appropriately matched analysis and synthesis filters. The following sections give the details.

\subsection{Restrictions on $M$ and $N$}

As opposed to fixed networks, aliasing components cannot be completely eliminated through cancellation in fully flexible FBR networks due to the large number of reallocation possibilities and constraints. Instead it must be possible to make them arbitrarily small in each channel which can be done using oversampling FBs and analysis filters with high enough stopband attenuation. To ensure this in the present setup, it is first observed that the filters are to extract spectra in accordance with Figures 2 and 5. This is achieved by dividing each granularity band into a number of uniform-band FB channels with principle filter magnitude responses according to Figure 6 (also cf. the discussion below). The filter bandwidths are thus $2 \pi / N$ and their transition bands are $2 \Delta$ wide. It is now required that passbands and transition bands of shifted terms caused by decimation do not overlap. This is achieved when

$$
M \leq \frac{N}{1+N \Delta / \pi}<N
$$

In addition to the constraint in (1), there is an additional relation between $M$ and $N$ that must be fulfilled and it is derived as follows. Through decimation and interpolation by the factor $M$, frequency shifts of $2 \pi m / M$ radians for $m=0,1, \ldots, M-1$ can be generated. It is required that one is able to generate all integer frequency shifts of the granularity frequency shift, that is, all frequency shifts $2 \pi q / Q$ for $q=0,1, \ldots, Q-1$. In particular, one must be able to shift the granularity bands by all values $2 \pi q / Q$. It is therefore required that $M$ be a multiple of $Q$, that is,

$$
M=B Q, \quad B \geq 1, B \text { integer. }
$$




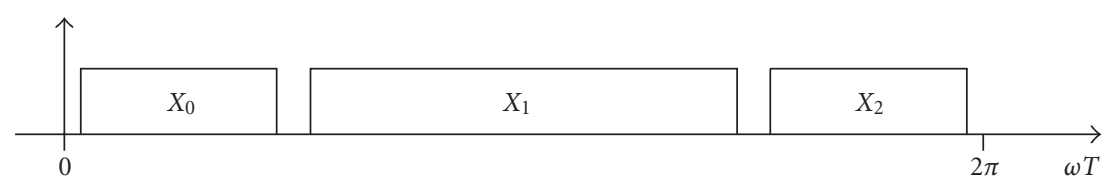

(a)

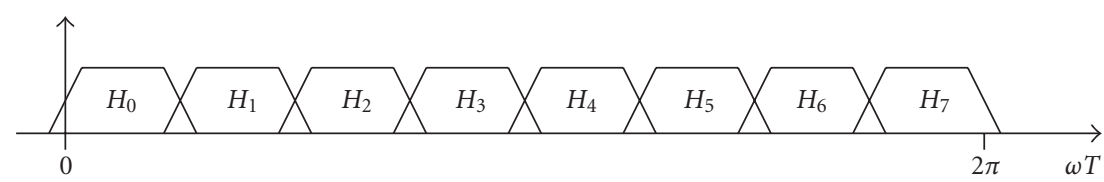

(b)

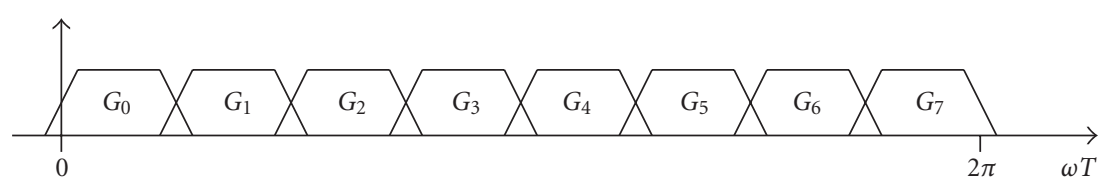

(c)

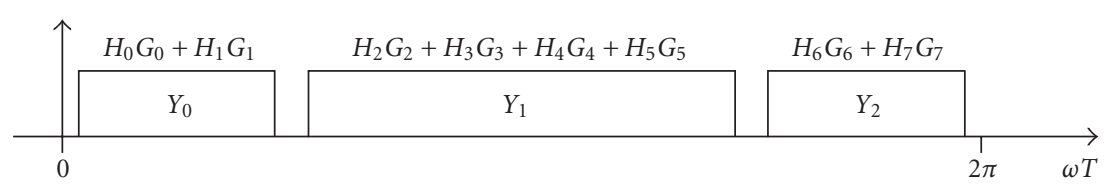

(d)

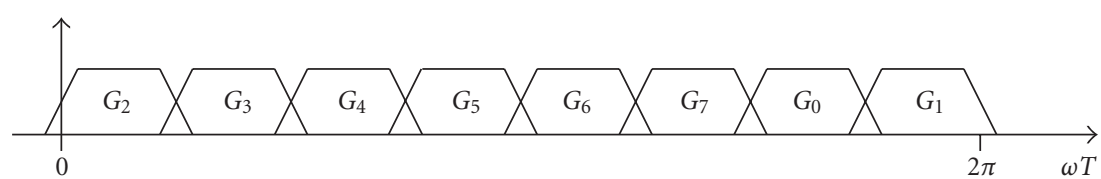

(e)

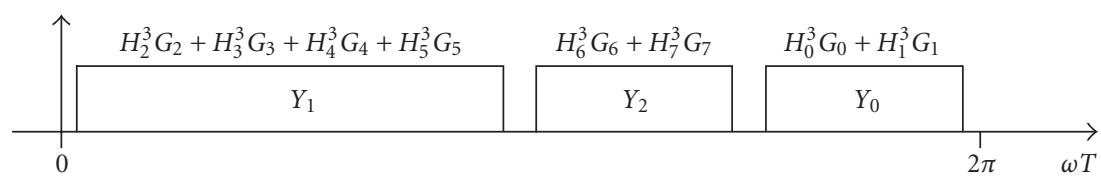

(f)

FIGURE 5: Illustration of frequency-band reallocation using the proposed FBs with $Q=4, N=8$. (a)-(d) Recombination of channels. (a), (b), (e), and (f) Recombination of channels and reallocation of subbands; $H^{3}$ stands for $H$ shifted three granularity-band shifts to the right which amounts to one shift to the left when $M=4$.

Since $N>M$ according to (1), this means that the number of uniform-band channels cannot equal the number of granularity bands. Instead, $N$ must be a multiple of $Q$, as illustrated in Figure 5. That is,

$$
N=A Q=\frac{A M}{B}, \quad A>B, A \text { integer. }
$$

Because the downsampling-by- $M$ blocks (upsampling-by- $M$ blocks) in Figure 4 can be propagated to the input (output) [9], the complexity for a given $N$ is minimized by selecting $M$ as large as possible without introducing aliasing, that is, without violating (1). Thus, it follows from (2) and (3) that
$B$ is selected as

$$
B=A-K, \quad 1 \leq K \leq A-1, K \text { integer }
$$

whereby

$$
M=N-K Q
$$

where $K$ is the smallest integer allowed without introducing aliasing. From (1) and (5), it follows that $K$ must satisfy

$$
K \geq \frac{\varepsilon N^{2}}{Q(Q+\varepsilon N)}=\frac{\varepsilon A^{2}}{1+\varepsilon A},
$$

where $\varepsilon$ denotes how much the guard band $2 \Delta$ occupies the 


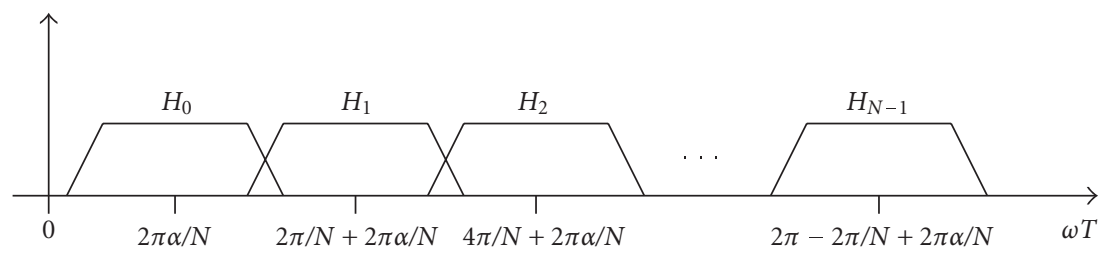

FIgure 6: Principle magnitude responses of the analysis filters.

granularity band $2 \pi / Q$, that is,

$$
2 \Delta=\varepsilon \frac{2 \pi}{Q}, \quad 0<\varepsilon \leq 1 .
$$

For any given $Q$, one can thus in principle choose any value of $N$ [which also determines $M$ through (5)] satisfying (2) and (3). In practice, it is selected so as to minimize the implementation complexity. This issue will be treated in Section 3.

\section{Discussion}

As seen in (3), the new network makes use of more FB channels than granularity bands (maximum number of user subbands). This is necessary in order to be able to generate all possible frequency shifts, the reason being that a slight oversampling is employed. At first sight, this may seem to be a drawback but is in fact an advantage in that the implementation complexity can be reduced by using more channels than the minimum one required by the application, which is $Q$ in the present application. It is possible to use $N>Q$ here (but not in all FB applications) because the role of the FB is to move spectra which one in principle can do with an arbitrary number of FB channels without degrading the performance in terms of BER, and so forth. In this way, the complexity may even be lower than that of regular maximally decimated FBs despite the fact that a slight oversampling is used.

\subsection{Analysis filters}

The analysis filters are obtained from a Dth-order linearphase FIR prototype filter with transfer function

$$
P(z)=\sum_{n=0}^{D} p(n) z^{-n}
$$

and with the impulse response $p(n)$ being symmetric, that is, $p(n)=p(D-n)$. The frequency response of such a prototype filter can be written as

$$
P\left(e^{j \omega T}\right)=e^{-j D \omega T / 2} P_{R}(\omega T),
$$

where $P_{R}(\omega T)$ is the real zero-phase frequency response [14] and $\omega T$ denotes the "discrete-time frequency." Its magnitude response is here principally as illustrated in Figure 7 . The analysis filters are complex-modulated versions of the prototype filter according to

$$
H_{k}(z)=\beta_{k} P\left(z W_{N}^{k+\alpha}\right), \quad k=0,1, \ldots, N-1,
$$

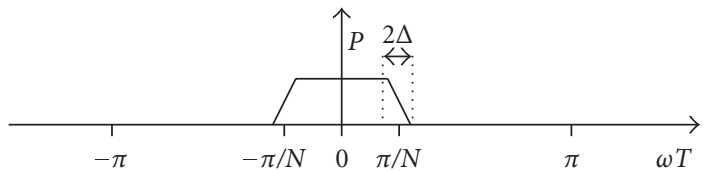

FIGURE 7: Principle magnitude response of the prototype filter.

where

$$
\begin{aligned}
& W_{N}=e^{-j 2 \pi / N}, \\
& \beta_{k}=W_{N}^{(k+\alpha) D / 2},
\end{aligned}
$$

and $\alpha$ is a real-valued constant used for placing the filters at the desired centre frequencies according to Figures 2 and 5.

The constants $\beta_{k}$ compensate for the phase rotations that generally are introduced when replacing the $D$ th-order linear-phase FIR filter $P(z)$ with $P\left(z W_{N}^{k+\alpha}\right)$. In this way, all analysis filters become linear-phase FIR filters with the same delay $(D / 2)$ as the prototype filter. Indeed, with $\beta_{k}$ as in (12), the frequency responses become

$$
H_{k}\left(e^{j \omega T}\right)=e^{-j D \omega T / 2} P_{R}\left(\omega T-\frac{2 \pi(k+\alpha)}{N}\right) .
$$

\subsection{Synthesis filters}

As opposed to conventional FBs, where one is interested in perfect reconstruction of the input $x(n)$, the synthesis filters must here be chosen in such a way that the outputs $y_{r}(n)$, $r=0,1, \ldots, q-1$, ideally are frequency-shifted (and delayed due to the FB delay) versions of the subsignals according to

$$
Y_{r}(z)=z^{-D} X_{r}\left(z W_{Q}^{s_{r}}\right),
$$

where $W_{Q}=e^{-j 2 \pi / Q}, 2 \pi / Q$ is the granularity frequency shift (minimum allowed frequency shift), and $s_{r}$ is an integer denoting the desired number of granularity-band shifts of subband $r$. For example, if it is desired to move $X_{0}\left(X_{2}\right)$ in Figure 2(b) to the position of $X_{2}\left(X_{0}\right)$, then $s_{r}=2\left(s_{r}=-2\right)$. Furthermore, it should be possible to approximate perfect FBR as close as desired (i.e., to approximate (14) as close as desired) for all values of $q, 1 \leq q \leq Q$, by properly designing the FB. Both of these criteria are met by selecting the synthesis filters as

$$
G_{k}(z)=\mu_{k r} H_{c_{k r}}(z),
$$


where

$$
\begin{gathered}
c_{k r}=k+A s_{r}, \\
\mu_{k r}=W_{N}^{\left(m_{r} N / M\right) D / 2}
\end{gathered}
$$

with

$$
m_{r}= \begin{cases}B s_{r}, & s_{r} \geq 0, \\ M+B s_{r}, & s_{r}<0,\end{cases}
$$

and $B$ being given by (4). The equations above hold for $k=$ $A i_{r}, A i_{r}+1, \ldots, A i_{r}+A n_{r}-1$, with $i_{r}$ denoting the left-most granularity band included in $x_{r}(n), A$ being given by (3), and $n_{r}$ denoting the number of granularity bands in subband $r$. To obtain (17), we have utilized that

$$
W_{M}^{m_{r}}=W_{N}^{m_{r} N / M}
$$

It should be noted here that the pair $(k, r)$ only takes on values that correspond to $c_{k r} \in[0, N-1]$ which for obvious reasons must be ensured. This will always be the case because our notations reflect the fact that the input subband $r$ covering the granularity-band positions $i$, for $i=i_{r}$, $i_{r}+1, \ldots, i_{r}+n_{r}-1$, is to be moved to the positions $i+s_{r}$. That is, it is a priori assumed that

$$
i_{r}, i_{r}+n_{r}-1 \in[0, Q-1]
$$

as well as

$$
i_{r}+s_{r}, i_{r}+s_{r}+n_{r}-1 \in[0, Q-1] .
$$

Since the number of FB channels is $N=A Q$, it follows that the input subband $r$ is also covered by the analysis FB channels $k$, for $k=A i_{r}, A i_{r}+1, \ldots, A i_{r}+A n_{r}-1$. For these values of $k$, it now follows from (20) that

$$
k+A s_{r} \in[0, A(Q-1)+A-1]=[0, N-1] .
$$

Thus, all $c_{k r}$ in (16) belong to [0,N-1].

The constants $\mu_{k r}$ compensate for the phase rotations that generally are introduced when replacing the $D$ th-order linear-phase FIR filters $H_{k}(z)$ with $H_{k}\left(z W_{M}^{m_{r}}\right)$. In this way, all synthesis filters become linear-phase FIR filters with the same delay $(D / 2)$ as the prototype filter (compare with the analysis filters in Section 2.4). Further simplifications are obtained by noting that it is always possible to make all $\mu_{k r}=1$. Indeed, we have

$$
\frac{m_{r} D}{2 M}=\text { integer } \Longrightarrow \mu_{k r}=1
$$

Thus, it is always possible to make all $\mu_{k r}$ equal to unity by selecting the filter order $D$ of the prototype filter properly. This is easily achieved by introducing a proper amount of additional delays.

Finally, it is noted that it follows from (15) that the network in Figure 3 with fixed filters and adjustable filters can be efficiently implemented by the network in Figure 4 that uses two sets of fixed filters and a variable channel switch.

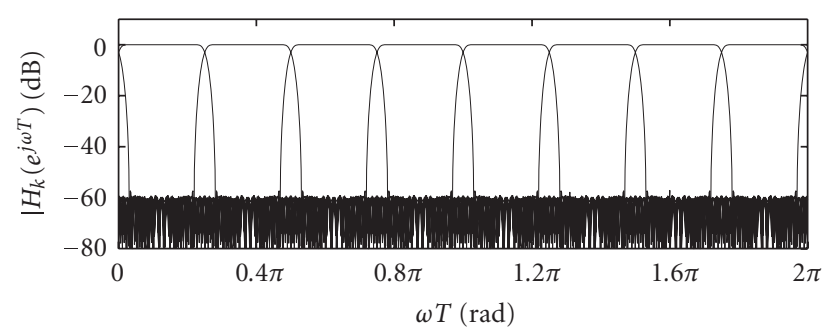

Figure 8: Analysis filters in Example 1.

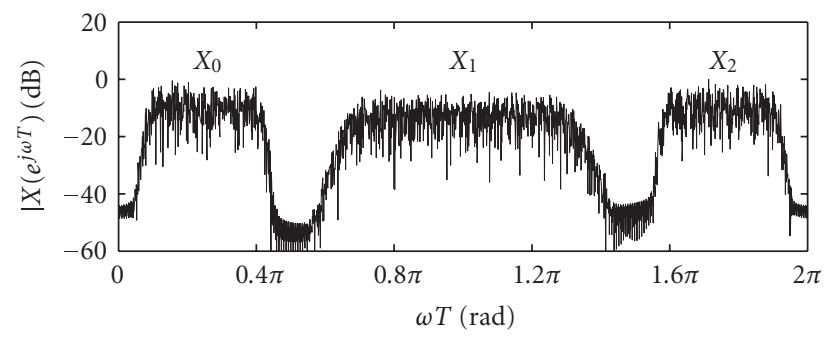

Figure 9: Input spectrum in Example 1.

Example 1. As a means of illustration, we consider the following example:

Number of granularity bands: $Q=4$

Number of FB channels: $\quad N=8$

Downsampling factor: $\quad M=4$

Transition band width: $\quad \Delta=0.125 \pi / Q=0.125 \pi / 4$

Frequency offset: $\quad \alpha=0.5$

Prototype filter order: $\quad D=134$

Number of subbands: $\quad q=3$

Number of granularity bands $n_{0}=1, n_{1}=2, n_{2}=1$

in each input subband:

First FB channel in each input subband:

$k_{0}=0, k_{1}=2, k_{2}=6$.

The magnitude responses of the analysis filters are shown in Figure 8. Design details will be discussed in Section 4. The input spectrum is plotted in Figure 9. We now consider three different reallocation schemes.

\section{Reallocation scheme (a)}

In this case, we assume that the output subband positions are the same as the input subband positions. This illustrates the ability of the filter bank to recombine several adjacent channels. In this case, the synthesis filters are the same as the analysis filters which means that the channel switch simply passes on its inputs as seen in Figure 13(a). The output spectrum becomes as shown in Figure 10. It is seen that it is the same as the input spectrum except for small errors introduced in the FBR network. By properly designing the network, these errors can be made negligible compared to other errors that are always present in communication systems. To exemplify: the input samples are in this example randomly generated quadrature amplitude modulated symbols (QAM-16) 


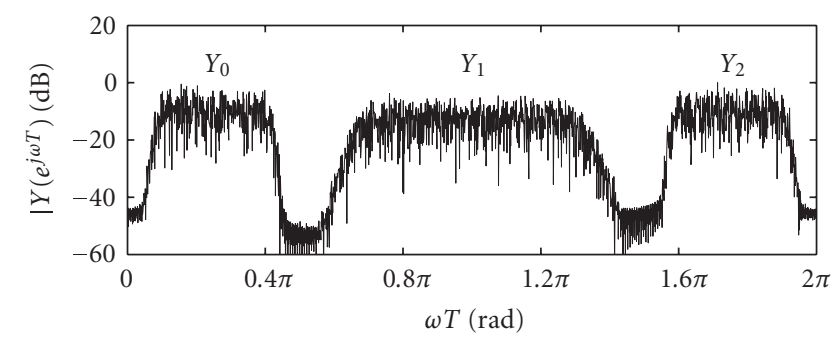

Figure 10: Output spectrum in Example 1 for reallocation scheme (a).

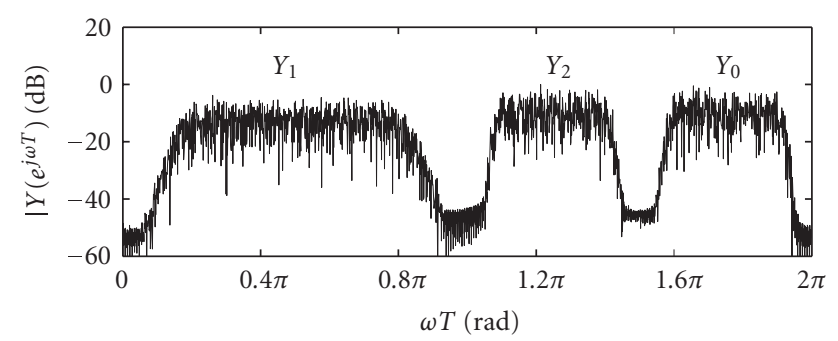

Figure 11: Output spectrum in Example 1 for reallocation scheme (b).

normalized so that the signal has a unity average power. Using an additional filter for recovering the first subband $\left(x_{0}\right)$, we find that the maximum distance between the input and output samples is below 0.01 . As a consequence, if the symbol error rate due to additive white noise alone (thus without errors created in the FBR network) is, say, $10^{-6}$, it will in the worst case be increased to $1.5 \times 10^{-6}$ due to the FBR network. By increasing the filter orders, and redesigning the FBR network, the degradation can be reduced to any level that in practice is negligible.

\section{Reallocation scheme (b)}

In this case, we assume a scheme as that shown earlier in Figures 5(a), 5(b), 5(e), and 5(f). This is achieved by selecting the synthesis filters according to (15) with the following numbers of granularity-band shifts: $s_{0}=3, s_{1}=s_{2}=-1$. These values imply that $m_{r}=3$, for $r=0,1,2$, which means that $\mu_{k r}=-j$ for all pairs of values $k r$ of interest in (17), that is, for $k r=00,10,21,31,41,51,62,72$. These values of $k r$ result in the following values of $c_{k r}: c_{00}=6, c_{10}=7, c_{21}=0$, $c_{31}=1, c_{41}=2, c_{51}=3, c_{62}=4, c_{72}=5$. When the synthesis FB is implemented using a switch and fixed filters, as shown in Figure 4, we recall that the role of the channel switch is to redirect its input at position $k$ to its output at position $c_{k r}$. In this example, the switch in Figure 4 is thus implemented as shown in Figure 13(b). The output spectrum becomes as shown in Figure 11. The errors are of the same order as in scheme (a).

\section{Reallocation scheme (c)}

In this case, we assume that the two narrow-band subbands are to interchange their positions as compared to scheme (b).

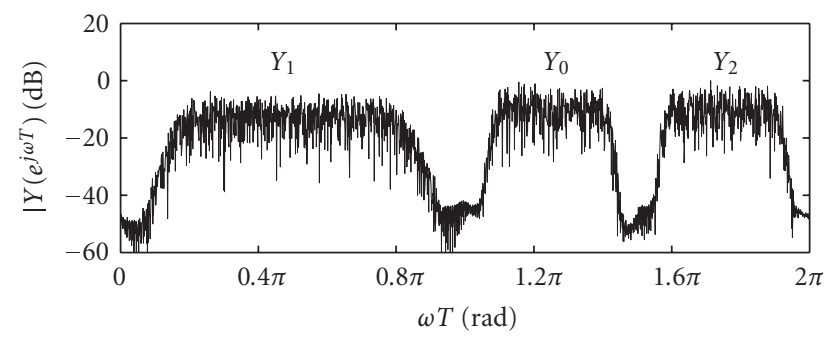

FIGURE 12: Output spectrum in Example 1 for reallocation scheme (c).

The output spectrum becomes in this case as shown in Figure 12. The errors are of the same order as in schemes (a) and (b). The parameter values are here as follows: $s_{0}=2$, $s_{1}=-1, s_{2}=0 ; m_{0}=2, m_{1}=-1, m_{2}=0 ; c_{00}=4$, $c_{10}=5, c_{21}=0, c_{31}=1, c_{41}=2, c_{51}=3, c_{62}=6$, $c_{72}=7 ; \mu_{00}=\mu_{10}=-1, \mu_{21}=\mu_{31}=\mu_{41}=\mu_{51}=-j$, $\mu_{62}=\mu_{72}=1$. The switch is in this case implemented as shown in Figure 13(c).

Finally, it is noted that we used a filter order of 134 in this example which resulted in multiplier values $\mu_{k r}$ not equal to unity. This was done in order to illustrate that the proposed technique works in such cases as well. By increasing the filter order to, for example, 136, all $\mu_{k r}$ become equal to unity.

\section{IMPLEMENTATION COMPLEXITY}

The main point of this section is the selection of the number of FB channels $N$ that minimize the overall implementation complexity when efficient DFT- and IDFT-based realizations are employed.

\subsection{Efficient DFT- and IDFT-based realizations}

Utilizing the polyphase form of $P(z)$ given by [9]

$$
P(z)=\sum_{i=0}^{N-1} z^{-i} P_{i}\left(z^{N}\right)
$$

where $P_{i}(z)$ are the polyphase components, $H_{k}(z)$ in (10) can be rewritten as

$$
H_{k}(z)=\beta_{k} \sum_{i=0}^{N-1} z^{-i} \alpha_{i} P_{i}\left(z^{N} W_{N}^{\alpha N}\right) W_{N}^{-k i},
$$

where

$$
\alpha_{i}=W_{N}^{-\alpha i}
$$

Making use of (25), well-known properties of DFT and IDFT FBs, and properties of downsamplers and upsamplers, it is now recognized that the analysis and synthesis FBs can be realized with the aid of an $N$-point IDFT and $N$-point DFT, respectively, as shown in Figures 14 and 15 where all arithmetic operations take place at the lowest sampling rate $\left(f_{\text {in }} / M\right)$. The multipliers in Figure 15 are given by

$$
\gamma_{k}=\beta_{k} W_{N}^{k}
$$




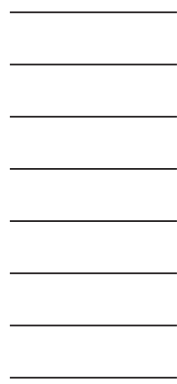

(a)

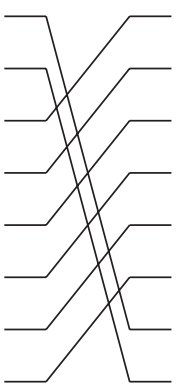

(b)

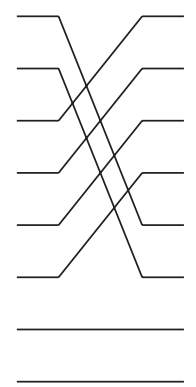

(c)
Figure 13: Channel switch in Example 1; Schemes (a), (b), and (c).

In the efficient synthesis FB in Figure 15, the separate outputs $y_{r}(n)$ from the channel combiner (Figure 3) are not available. This means that the multipliers $\mu_{k r}$ have to be placed at the input, preferably in front of the DFT (instead of the channel switch) since they can then be combined with the multipliers already present there; this is illustrated in Figure 15. In this way, the multiplier cost can be minimized also in those cases when $\mu_{k r} \neq 1$. It should also be noted that not having the separate outputs $y_{r}(n)$ available is not a problem in the SISO case as only the composite output $y(n)$ is supposed to be used here. However, in the MIMO case, this is a problem that must be taken care of (see Section 5).

In summary, it is seen that the proposed FBR network has about the same low complexity as that of a regular fixed modulated FB but with the additional inherent flexibility. Naturally, there is an overhead cost due to the channel switch, but such a block is required in all flexible FBR networks and thus not an extra cost in comparison with other such networks.

\subsection{Selection of $N$ that minimizes the implementation complexity}

As seen earlier in Section 2.3, there is not just one selection of the number of FB channels $N$ that can be used for a fixed prespecified number of subbands $Q$ and guard band width $2 \Delta$. In practice, it is of course of interest to select $N$ so that the overall implementation complexity is minimized. This issue is treated in this section.

Because the prototype filter $P(z)$ is a linear-phase FIR filter, its order $D$ can be estimated as [15]

$$
D=\frac{K_{p}}{2 \Delta}
$$

where $2 \Delta$ is the transition bandwidth (which equals the width of the guard band, see Figure 2) and

$$
K_{p}=\frac{-20 \log _{10}\left(\sqrt{\delta_{c} \delta_{s}}\right)-13}{14.6 /(2 \pi)}
$$

with $\delta_{c}$ and $\delta_{s}$ being the passband and stopband ripples, respectively. The order is thus inversely proportional to the transition bandwidth. The number of multipliers required in the prototype filter is $D+1$, since the symmetry of the linear-phase FIR prototype filter cannot be utilized. Further, the implementation of an $N$-point DFT, as well as an IDFT, requires about $0.5 \mathrm{~N} \log _{2}(N)$ multiplications per block of $N$ input/output samples, provided that an efficient FFT algorithm is used. The complexity $C_{A}$ of the analysis FB becomes thereby ${ }^{1,2}$

$$
\begin{aligned}
C_{A} & =\frac{D+1+0.5 N \log _{2}(N)}{M} \\
& =\frac{K_{P} / \Delta+2+N \log _{2}(N)}{2 M} .
\end{aligned}
$$

For a fixed $N$, it is evident from (30) that the complexity reduces as $M$ increases. This justifies the choice $M=N-K Q$ in (5) in Section 2.3. Expressed in terms of $A$, with $N=A Q$ according to $(3),(30)$ can alternatively be written as

$$
C_{A}=\frac{K_{P} / \Delta+2+A Q \log _{2}(A Q)}{2 M} .
$$

Assuming that equality holds in (1) and (6), one may find the minimum of the function $C_{A}$ by setting its derivative with respect to $A$ to zero and solve for $A$ yielding the optimum $A$, denoted here as $A_{\text {opt }}$. However, since $C_{A}$ and its derivative involve both $A$ and the logarithm of $A$, it is not possible to express $A_{\text {opt }}$ in a simple form. In practice it is therefore advisable to plot $C_{A}$ as a function of $A$ from which $A_{\text {opt }}$ easily can be identified. This is illustrated in Figure 16 for two different values of $K_{P}$. One should note here that there are basically three different cases that may occur. In the first case, as seen in the uppermost plot in Figure 16, $A_{\text {opt }}$ lies between $A_{\text {min }}$ and $A_{\max }$, which denote the minimum and maximum values of $A$, respectively. The minimum value is always $A_{\min }=2$ due to (2) and (3). The maximum value is determined by the upper bound on $N$ that exists because the number of channels $(N / Q)$ in each subband times the guard bandwidth $(2 \Delta)$ cannot exceed the granularity bandwidth $(2 \pi / Q) .{ }^{3}$ Hence, $N$ is bounded by

$$
N \leq \frac{\pi}{\Delta}=\frac{Q}{\varepsilon},
$$

where the equality comes from (7). This implies that the maximum value of $A$ is

$$
A_{\max }=\left\lfloor\frac{1}{\varepsilon}\right\rfloor
$$

where $\lfloor x\rfloor$ stands for the maximum integer smaller than or equal to $x$. In the second case, as seen in the downmost plot in Figure $16, A_{\text {opt }}=A_{\max }$. This occurs when $K_{P}$ is large. In the third case, $A_{\mathrm{opt}}=A_{\mathrm{min}}$, which occurs when $K_{P}$ is small.

\footnotetext{
${ }^{1}$ As a measure of complexity, the multiplication rate is used. It is here the number of multiplications per input (output) sample in the analysis FB (synthesis FB). The multiplication rate takes into account the data rate at which the multiplications are performed.

2 The number of additions and delay elements is here roughly proportional to the number of multiplications and is therefore omitted in the discussion.

3 The bound can be increased, in principle to infinity, by reducing the guard bandwidth, but this does not make sense as it will increase the filter order.
} 


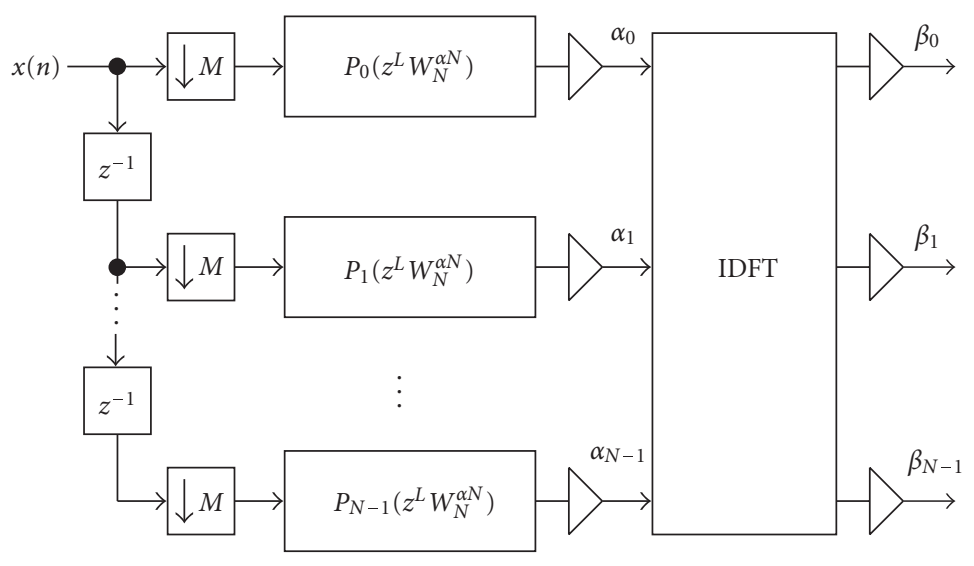

Figure 14: Analysis FB realizing the analysis filters $H_{k}(z)$, as given by $(10)$, where $L=A / B=$ integer. When $A / B$ is not an integer, a more general polyphase implementation of the polyphase components $P_{i}\left(z^{N}\right)$ followed by downsampling has to be used [9], but all filtering operations can still be moved to the input rate.

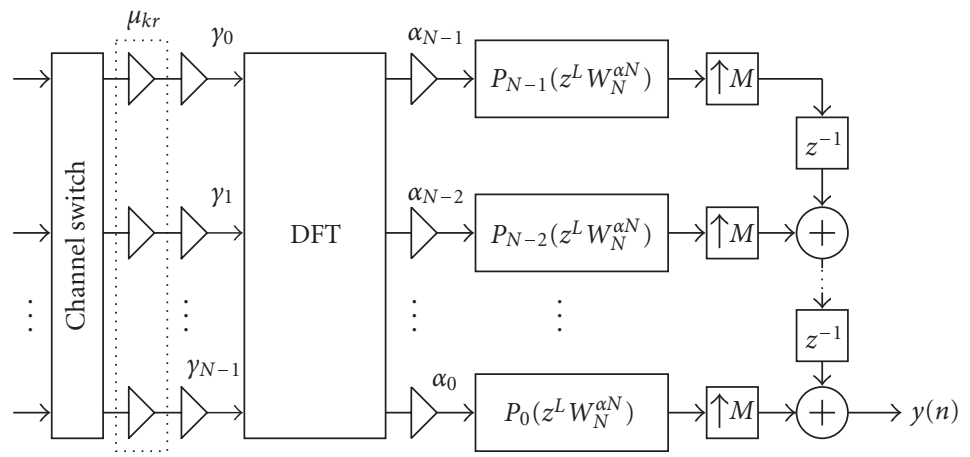

Figure 15: Synthesis FB realizing the synthesis filters $G_{k}(z)$ as given by (15) using a channel switch and fixed filters $H_{k}(z)$ as given by $(10)$.

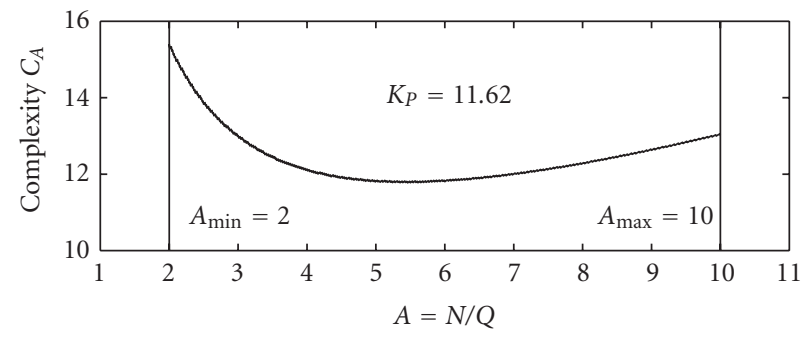

(a)

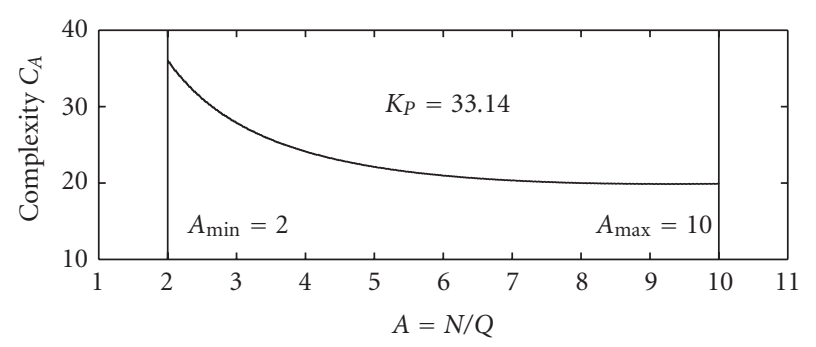

(b)

Figure 16: Complexity $C_{A}$ as a function of $A=N / Q$ for two different values of $K_{P}$ as given by (29).
In the discussion above, some simplifications were made in order to arrive at the optimum $A$. In practice, there are several issues that must be taken into consideration which complicates the minimization of the complexity. These issues are discussed below.

First, it was assumed that the passband and stopband ripples are constant regardless the value of $N$. As $N$ increases, one should rather replace the stopband ripple $\delta_{s}$ by $\delta_{s} / N$ though, to compensate for the larger number of aliasing components, at least when using worst-case design techniques (see (49) in Section 4.3). However, since the order of an FIR filter depends on the stopband ripple logarithmically, this compensation will have a minor effect upon the order. Hence, if we instead use $\delta_{s} / N$ above, the complexity $C_{A}$ as a function of $A$ will only change slightly.

Second, it was assumed that the prototype filter is a regular lowpass linear-phase FIR filter without requirements in the transition band. However, one should compensate for the fact that the prototype filter must exhibit an approximately power complementary behavior in the transition band. This means that the constant $K_{P}$ in (28) should be replaced by $c K_{P}, c>1$. Our experience is that $c$ is approximately constant, regardless of the other parameter values, although there exist no empirically derived formulas based on a large number of 
designs that confirm this assertion. If $c$ is constant, the effect is that we simply increase the value of $K_{P}$, the result of which is that $A_{\text {opt }}$ will move closer to $A_{\max }$, unless $A_{\text {opt }}=A_{\max }$ for $K_{P}$ in which case $A_{\text {opt }}$ remains the same. This is seen in Figure 16.

Third, we have assumed that all multiplications have the same cost in an implementation. However, in cases where $\alpha$ takes on the value $0, \pm 0.25$, or \pm 0.5 (implying that $W_{N}^{-\alpha N}$ takes on the values $1, \pm j$, and -1 ) each multiplication in the polyphase components only requires one real multiplication whereas the multiplications in the DFT and IDFT, most of which are always complex, require at least three real multiplications [16]. Taking this into account amounts to replacing 0.5 in (30) with 1.5 , the result of which is that $A_{\text {opt }}$ will move closer to $A_{\text {min }}$, unless $A_{\text {opt }}=A_{\text {min }}$ for the value 0.5 , in which case $A_{\text {opt }}$ remains the same.

Taking these issues into account, one can thus still generate a plot as that in Figure 16 from which the optimum value of $A$ can be determined. As to the synthesis FB, its complexity is the same as that of the analysis FB when all $\mu_{k r}$ equal unity, which always can be guaranteed if a certain amount of additional delay can be accepted. In the most general case, with some or all of $\mu_{k r}$ not being equal to unity, at most $N / M$ additional complex multiplications per input/output sample are required. Since $N / M$ never exceeds $1 / 2$, this is a minor extra cost during normal operation.

\section{DESIGN}

This section considers the design of the flexible FBR network which amounts to determining the linear-phase FIR prototype filter $P(z)$ so that the network approximates perfect FBR. This is in principle the same design problem as in conventional FBs, but it is much more complex here due to the many different reallocation schemes involved.

\subsection{Distortion and aliasing}

Using well-known input-output relations for the downsampler and upsampler [9], one finds that the $z$-transform of the output $y(n)$ in Figures 3 and 4 can be expressed as

$$
Y(z)=\sum_{r=0}^{q-1} Y_{r}(z)
$$

where the outputs $y_{r}(n), r=0,1, \ldots, q-1$, are given by

$$
Y_{r}(z)=\sum_{m=0}^{M-1} V_{r m}(z) X\left(z W_{M}^{m}\right)
$$

with $W_{M}=e^{-j 2 \pi / M}$ and

$$
V_{r m}(z)=\sum_{k=k_{r}}^{k_{r}+A n_{r}-1} H\left(z W_{M}^{m}\right) G_{k}(z),
$$

where $k_{r}=A i_{r}$ denotes the first FB channel included in the same band as $x_{r}(n)$. We now wish to state the condition under which perfect FBR is obtained. In order to do that in a simple form, we first recognize that (14) in the Fourier domain corresponds to

$$
Y_{r}\left(e^{j \omega T}\right)=e^{-j D \omega T} X_{r}\left(e^{j \omega T} W_{Q}^{s_{r}}\right)
$$

which, equivalently, can be written as

$$
Y_{r}\left(e^{j \omega T}\right)=F_{r}\left(e^{j \omega T} W_{Q}^{s_{r}}\right) X\left(e^{j \omega T} W_{Q}^{s_{r}}\right)
$$

with

$$
F_{r}\left(e^{j \omega T}\right)= \begin{cases}e^{-j D \omega T}, & \omega T \in \Omega_{x}^{(r)}, \\ 0, & \omega T \notin \Omega_{x}^{(r)},\end{cases}
$$

where

$$
\begin{aligned}
\Omega_{x}^{r}=[ & \left(2 i_{r}-1\right) \pi / Q+2 \pi \alpha / Q+\Delta, \\
& \left.\left(2 i_{r}+2 n_{r}-1\right) \pi / Q+2 \pi \alpha / Q-\Delta\right]
\end{aligned}
$$

and $1 / T$ is the input and output sampling rate.

The network is a perfect FBR network if the right-hand side of (35) for $z=e^{j \omega T}$ equals that in (38). Thus, the network is a perfect FBR network if $V_{r m}(z)$ in (36) for all $r$ and $m$ satisfy

$$
\begin{gathered}
V_{r m}\left(e^{j \omega T}\right)=F_{r}\left(e^{j \omega T} W_{Q}^{s_{r}}\right), \quad m=m_{r}, \\
V_{r m}(z)=0, \quad m \neq m_{r},
\end{gathered}
$$

where $F_{r}\left(e^{j \omega T}\right)$ is given by (39) and $m_{r}$ is given by (18). We have also utilized that $W_{Q}^{s_{r}}=W_{M}^{m_{r}}$. When $s_{r}$ is negative, $m_{r}$ equals $M+B s_{r}$ instead of $B s_{r}$ which is due to the fact that only positive values of $m$ are used in (35). It is possible to replace $B s_{r}$ with $M+B s_{r}$ because $W_{M}^{m}=W_{M}^{M+m}$.

It should be noted that for the special case with $q=Q=$ 1 , a regular FB is obtained. In this case, no reallocation can take place (since only one band is present) and the whole band should be reconstructed. In this special case, a perfect FBR is the same as a perfect reconstruction FB.

\subsection{Relation between $V_{r m_{r}}\left(e^{j \omega T}\right)$ and $V_{r 0}\left(e^{j \omega T}\right)$}

This section shows that the FBR network for all $s_{r}$ of interest can be related to an FBR network with $s_{r}=0$, that is, when subbands are not reallocated but only recombined. This amounts to showing that $V_{r m_{r}}\left(e^{j \omega T}\right)$ are frequency shifted versions of $V_{r 0}\left(e^{j \omega T}\right)$. This relation eases the design substantially as discussed in the following section.

We first note that the frequency responses corresponding to $H_{k}\left(z W_{M}^{m_{r}}\right)$ and $G_{k}(z)$ are obtained from (9), (10), and (15), as

$$
\begin{aligned}
H_{k}\left(e^{j \omega T} W_{M}^{m_{r}}\right)= & e^{-j D \omega T / 2} W_{N}^{-\left(m_{r} N / M\right) D / 2} \\
& \times P_{R}\left(\omega T-\frac{2 \pi\left(k+m_{r} N / M+\alpha\right)}{N}\right), \\
G_{k}\left(e^{j \omega T}\right)= & e^{-j D \omega T / 2} W_{N}^{\left(m_{r} N / M\right) D / 2} \\
& \times P_{R}\left(\omega T-\frac{2 \pi\left(k+m_{r} N / M+\alpha\right)}{N}\right),
\end{aligned}
$$


respectively. Hence, the frequency responses corresponding to $V_{r m_{r}}(z)=H_{k}\left(z W_{M}^{m_{r}}\right) G_{k}(z)$ become

$$
\begin{aligned}
& V_{r m_{r}}\left(e^{j \omega T}\right) \\
& \quad=e^{-j D \omega T} \sum_{k=k_{r}}^{k_{r}+A n_{i}-1} P_{R}^{2}\left(\omega T-\frac{2 \pi\left(k+m_{r} N / M+\alpha\right)}{N}\right) .
\end{aligned}
$$

Thus, the distortion function is a linear-phase function with delay $D$ and magnitude

$$
\left|V_{r m_{r}}\left(e^{j \omega T}\right)\right|=\sum_{k=k_{r}}^{k_{r}+A n_{i}-1} P_{R}^{2}\left(\omega T-\frac{2 \pi\left(k+m_{r} N / M+\alpha\right)}{N}\right) .
$$

We note that

$$
V_{r m_{r}}\left(e^{j \omega T}\right)=V_{r 0}\left(e^{j \omega T} W_{N}^{m_{r} N / M}\right),
$$

where $V_{r 0}\left(e^{j \omega T}\right)$ is given by

$$
V_{r 0}\left(e^{j \omega T}\right)=e^{-j D \omega T} \sum_{k=k_{r}}^{k_{r}+A n_{i}-1} P_{R}^{2}\left(\omega T-\frac{2 \pi(k+\alpha)}{N}\right),
$$

is the distortion function when the subbands are only recombined (thus not reallocated). This shows that $V_{r m_{r}}(z)$ are frequency-shifted versions of $V_{r 0}(z)$. Hence, if the network is a near-perfect FBR network when $G_{k}(z)=H_{k}(z)$, so is the network when these $G_{k}(z)$ are replaced with the functions in (15). It should be mentioned, however, that the aliasing components do not remain the same but their magnitudes are still bounded by the stopband attenuation of the prototype filter.

\subsection{Minimax design}

Filter banks are commonly designed using minimax or leastsquares design techniques, or combinations of such design techniques [17]. This paper discusses minimax design but the alternatives can of course be used as well after appropriate modifications.

Due to (45), it suffices to control $V_{r 0}\left(e^{j \omega T}\right)$, given by (46), for $r=0,1, \ldots, q-1$, and the aliasing terms in the design. For this reason, let the specifications of $V_{r m}(z)$ be

$$
\left|V_{r 0}\left(e^{j \omega T}\right)-F_{r}\left(e^{j \omega T}\right)\right| \leq \delta_{0}, \quad \omega T \in[0, \pi],
$$

where $\delta_{0}>0$ and $F_{r}\left(e^{j \omega T}\right)$ is given by (39), and

$$
\left|V_{r m}\left(e^{j \omega T}\right)\right| \leq \delta_{1}, \quad \omega T \in[0, \pi],
$$

for $m=0,1, \ldots, M-1, m \neq m_{r}, m_{r}$ being given by (18), and $\delta_{1}>0$. The parameters $\delta_{0}$ and $\delta_{1}$ are prescribed distortion and aliasing errors, respectively, and determined by the application at hand. In conventional FBs, the distortion and aliasing errors can be made zero by using certain classes of PR FBs. It is however not likely that one can find practical realizations with zero distortion and aliasing errors when it comes to flexible FBR reallocation networks. The reason is that (41) should be satisfied for all $r=0,1, \ldots, q-1$, all $q=0,1, \ldots, Q-1$, and all feasible combinations and reallocations schemes. This means that the number of conditions to satisfy is substantially larger for flexible FBR networks than for regular FBs. Therefore, one has to accept the use of nearperfect FBR networks. This is however not really a problem because the FB is to be used in a communication system which always contains other sources of errors which together result in a certain BER. The important point is that it is possible to design the FBR network to approximate perfect FBR as close as desired as one thereby can make the degradation due to the imperfect FBR network negligible compared to the other errors involved. In addition, it is known that the use of near-PR FBs instead of PR FBs can reduce the complexity substantially [17] which means that one should aim for near-PR systems anyhow. Exactly how close to perfect FBR the network must be is not specific for the proposed network but depends on the communication environment, modulation techniques, and other factors [18] that are beyond the scope of this paper.

In principle, one can apply any standard nonlinear optimization technique [19] directly to meet the criteria in (47) and (48). However, as the optimization is nonlinear, and will contain many constraints, it may become numerically difficult or infeasible to solve this problem in practice. One way to reduce the number of constraints substantially is to allow a slight overdesign and replace (48) with

$$
\left|P\left(e^{j \omega T}\right)\right| \leq \frac{\delta_{1}}{N}, \quad \omega T \in \Omega_{s},
$$

where $\Omega_{s}$ denotes the stopband of $P(z)$. It is also noted that nonlinear optimization benefits from a good initial solution which here can be obtained by using the well-known algorithm in [20] which generates linear-phase FIR filters optimum in the minimax sense.

Finally, we note that, for a fixed reallocation scheme, (47) and (48) correspond to the requirements of partially reconstructing FBs [7]. However, as already explained, the design problem is much more complex here as a large number of reallocations options must be handled simultaneously in the design.

\section{FLEXIBLE FBR MIMO NETWORKS}

This section shows how to generalize the proposed SISO networks to MIMO networks.

\section{1. $K$-input $K$-output frequency-band reallocation networks}

Generalizing the SISO system considered so far to a MIMO system with equal number $(K)$ of inputs and outputs, we propose the flexible FBR network depicted in Figure 17. It is here assumed that the subbands are reallocated to unique positions. Further, the analysis FBs (AFBs) and synthesis FBs (SFBs) are instances of the fixed FBs used in Section 3. Thus, the only difference from the SISO case is that the channel 


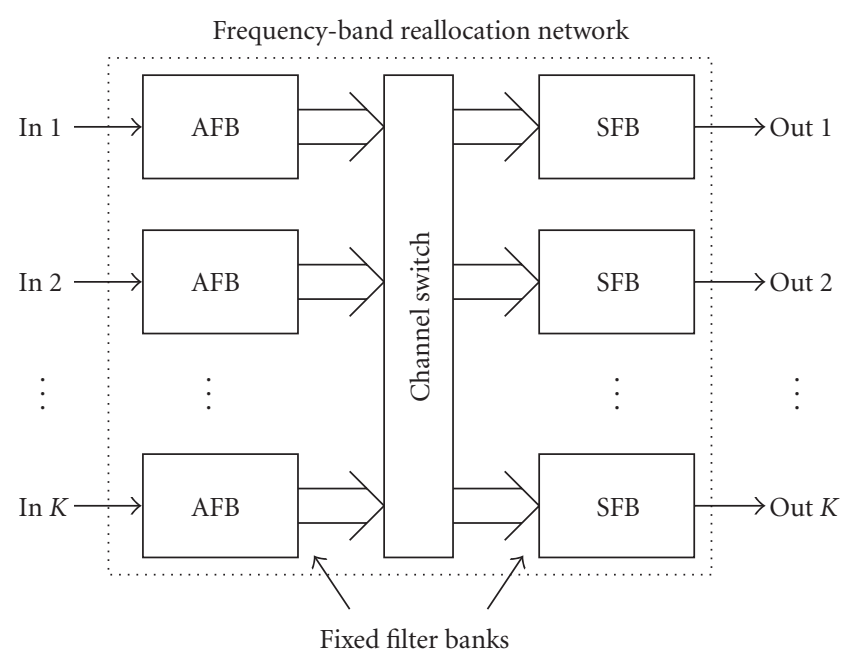

FIGURE 17: Proposed $K$-input $K$-output flexible FBR network using fixed FBs and a channel switch.

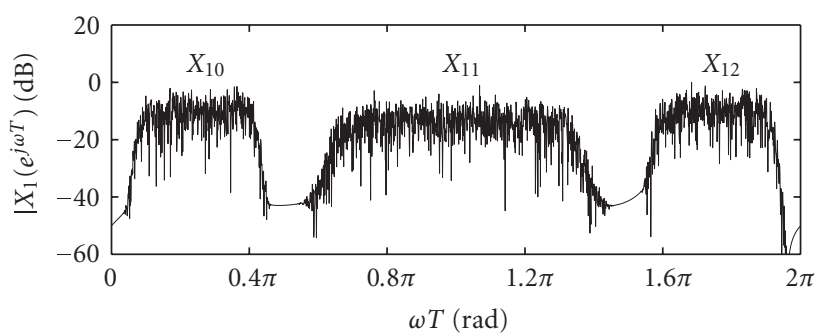

FIgURE 18: Input 1 spectrum in Example 2.

switch in this MIMO case is able to redirect information from any input beam to any output beam, as illustrated in the example below. If the FBR SISO network is designed as outlined in Section 4, the overall performance for each output subband in the MIMO network will be the same as in the SISO network, except for some minor negligible differences caused by differences in the aliasing terms. Consequently, it suffices to design one prototype filter for the SISO case, that is, as outlined in Section 4, and then use $K$ instances of the corresponding FBs according to Figure 17. This implies that the proposed MIMO system is modular which is attractive from the design and implementation points of view.

Example 2. The function of the proposed FBR MIMO network is illustrated through an example with two input and output beams. The two input spectra are plotted in Figures 18 and 19. It is desired to reallocate the subbands according to Figures 20 and 21, which plot the two output spectra. The frequency-band reallocation is achieved by using the channel switch in Figure 22 and FBs with the same filter magnitude responses as used earlier in Example 1 (Figure 8), but with an additional filter delay introduced to make all $\mu_{k r}$ equal to unity.

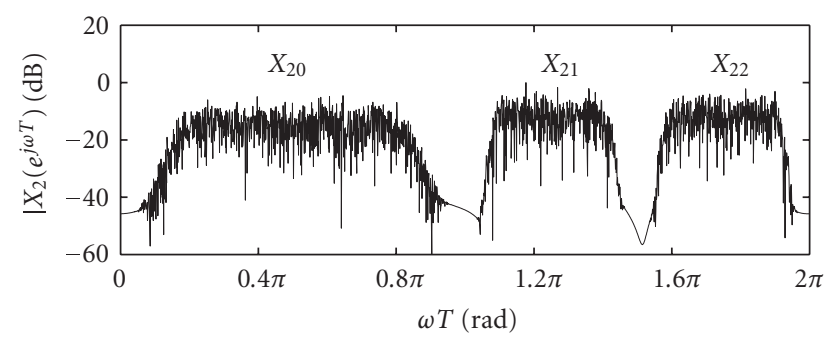

FIGURE 19: Input 2 spectrum in Example 2.

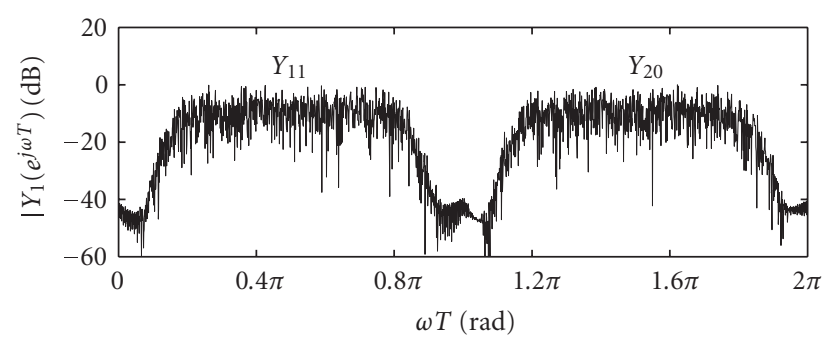

FIgURE 20: Output 1 spectrum in Example 2.

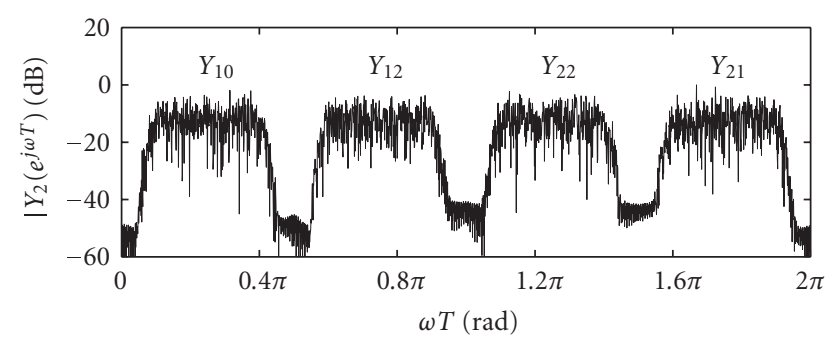

FIgURE 21: Output 2 spectrum in Example 2.

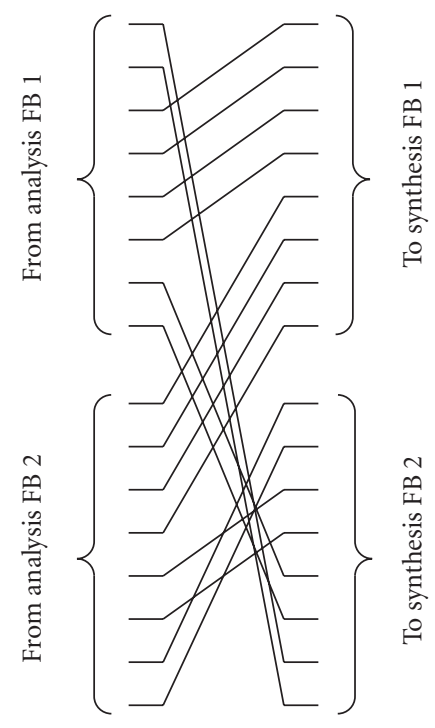

FIGURE 22: Channel switch in Example 2. 


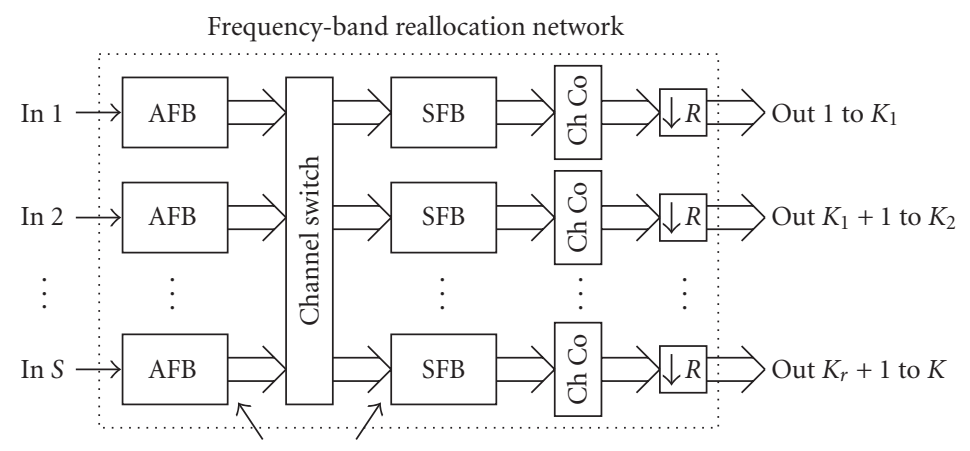

Fixed filter banks

Figure 23: Proposed $S$-input $K$-output FBR network using fixed FBs, a channel switch, and channel combiners (Ch Co).

\subsection{S-input K-output systems}

Generalizing the $K$-input $K$-output system considered above to an $S$-input $K$-output system, we propose the flexible FBR network depicted in Figure 23. Again, it is assumed that the subbands are reallocated to unique positions which implies that $K \geq S$. It is further assumed that

$$
K=R S
$$

which corresponds to the fact that the output beams' bandwidth is assumed to be $R$ times narrower than that of the input beams ${ }^{4}$. This means that only some of the synthesis FB outputs are combined to form the outputs. It also means that decimation by $R$ can take place at the outputs without introducing aliasing. Hence, in principle, it is again possible to use only $S$ fixed synthesis FBs, but it is then not possible to directly redirect all output subbands to the baseband. Instead, one has to make use of the whole band and let the subsequent decimation make the mapping to the baseband; that is, the spectrum at the input of each decimator has a bandwidth of $\pi / R$ and is positioned between $p \pi / R$ and $(p+1) \pi / R$ with respect to the input sampling rate, with $p$ being an integer belonging to the set $[0, R-1]$.

However, a problem of using only $S$ fixed synthesis FBs is that it is then not possible to make use of the efficient realization in Figure 15 because the outputs of the synthesis filters are not available in that structure. To get around this problem, we propose to use instead $K=R S$ fixed synthesis FBs, each being an instance of the fixed synthesis FB used in Section 3 (Figure 15) but with some of the inputs to the DFT being zero which corresponds to the fact that only a subset of the FB channels will be utilized in each synthesis

\footnotetext{
${ }^{4}$ This case can be generalized to allow outputs with different data rates which amounts to allowing different downsampling factors at the output in Figure 23. In the implementation, different instances of synthesis FBs must then be used, with different numbers of inputs to the DFT being set to zero.
}

FB (See Footnote 4). In this case, one can redirect all output subbands to the baseband. Further, by making use of multirate identities [9] one can make the overall computational complexity of the $K$ synthesis FBs roughly the same as earlier. That is, the number of arithmetic operations per time unit remains the same whereas the number of synthesis FB instances is $R$ times higher. Note that analysis FBs can be implemented in the same way as for the SISO case and MIMO case with equal number of inputs and outputs. It is thus only the synthesis parts that need to be modified in this generalized MIMO case.

\subsection{Further generalizations}

One may also think of allowing $S>K$ in the network in Section 5.2 above. However, this requires synthesis FBs with upsampling rates higher than the downsampling rates used in the analysis FBs. The proposed network cannot be used for this case straightforwardly and is therefore not discussed further in this paper.

\section{CONCLUDING REMARKS}

This paper introduced a new class of flexible FBR networks using variable oversampled complex-modulated FBs. The new network can outperform existing ones when all the aspects flexibility, low complexity and inherent parallelism, near-perfect FBR, and simplicity are considered simultaneously. The paper discussed design and complexity issues and provided examples that demonstrated the functionality. Finally, we wish to make the following two remarks. First, the FB prototype filter used in this paper is a linear-phase FIR filter. It is possible to use instead a nonlinear-phase FIR filter or an IIR filter, after appropriate modifications, as a means to reduce the delay and/or the implementation complexity. Second, the proposed design technique is simple, and attractive in that sense, but it generates overdesigned FBs. There is thus room for reduction of the complexity by using other design methods. These are topics for future research. 


\section{REFERENCES}

[1] B. Arbesser-Rastburg, R. Bellini, F. Coromina, et al., "R\&D directions for next generation broadband multimedia systems: an ESA perspective," in Proceedings of 20th AIAA International Communication Satellite Systems Conference and Exhibit, Montreal, Quebec, Canada, May 2002.

[2] E. Del Re and L. Pierucci, "Next-generation mobile satellite networks," IEEE Communications Magazine, vol. 40, no. 9, pp. 150-159, 2002.

[3] M. Wittig, "Satellite onboard processing for multimedia applications," IEEE Communications Magazine, vol. 38, no. 6, pp. 134-140, 2000.

[4] M.-L. Boucheret, I. Mortensen, and H. Favaro, "Fast convolution filter banks for satellite payloads with on-board processing," IEEE Journal on Selected Areas in Communications, vol. 17, no. 2, pp. 238-248, 1999.

[5] G. Chiassarini and G. Gallinaro, "Frequency domain switching: algorithms, performances, implementation aspects," in Proceedings of the 7th Tyrrhenian International Workshop on Digital Communications, Viareggio, Italy, September 1995.

[6] H. G. Göckler and B. Felbecker, "Digital on-board FDMdemultiplexing without restrictions on channel allocation and bandwidth," in Proceedings of the 7th International Workshop on Digital Signal Processing Techniques for Space Applications (DSP '99), Noordwijk, The Netherlands, 1999.

[7] T. Q. Nguyen, "Partial spectrum reconstruction using digital filter banks," IEEE Transactions on Signal Processing, vol. 41, no. 9, pp. 2778-2795, 1993.

[8] W. A. Abu-Al-Saud and G. L. Stuber, "Efficient wideband channelizer for software radio systems using modulated PR filterbanks," IEEE Transactions on Signal Processing, vol. 52, no. 10, part 1, pp. 2807-2820, 2004.

[9] P. P. Vaidyanathan, Multirate Systems and Filter Banks, Prentice-Hall, Englewood Cliffs, NJ, USA, 1993.

[10] P. N. Heller, T. Karp, and T. Q. Nguyen, "A general formulation of modulated filter banks," IEEE Transactions on Signal Processing, vol. 47, no. 4, pp. 986-1002, 1999.

[11] T. Karp and N. J. Fliege, "Modified DFT filter banks with perfect reconstruction," IEEE Transactions on Circuits and Systems II: Analog and Digital Signal Processing, vol. 46, no. 11, pp. 1404-1414, 1999.

[12] J. Alhava, A. Viholainen, and M. Renfors, "Efficient implementation of complex exponentially-modulated filter banks," in Proceedings of IEEE International Symposium on Circuits and Systems (ISCAS '03), vol. 4, pp. 157-160, Bangkok, Thailand, May 2003.

[13] H. Johansson and P. Löwenborg, "Flexible frequency-band reallocation network based on variable oversampled complexmodulated filter banks," in Proceedings of IEEE International Conference on Acoustics, Speech and Signal Processing (ICASSP '05), vol. 3, pp. 973-976, Philadelphia, Pa, USA, March 2005.

[14] T. Saramäki, "Finite impulse response filter design," in Handbook for Digital Signal Processing, S. K. Mitra and J. F. Kaiser, Eds., chapter 4, pp. 155-277, John Wiley \& Sons, New York, NY, USA, 1993.

[15] J. F. Kaiser, "Nonrecursive digital filter design using $I_{0}$-sinh window function," in Proceedings of IEEE International Symposium on Circuit and Systems (ISCAS '74), pp. 20-23, San Francisco, Calif, USA, April 1974.
[16] K. K. Parhi, VLSI Digital Signal Processing Systems: Design and Implementation, chapter 8, John Wiley \& Sons, New York, NY, USA, 1999.

[17] T. Saramäki and R. Bregovic, "Multirate systems and filter banks," in Multirate Systems: Design and Applications, G. Jovanovic-Dolecek, Ed., chapter 2, pp. 27-85, Idea Group, Hershey, Pa, USA, 2002.

[18] S. Haykin, Digital Communications, John Wiley \& Sons, New York, NY, USA, 1988.

[19] S. G. Nash and A. Sofer, Linear and Nonlinear Programming, McGraw-Hill, New York, NY, USA, 1996.

[20] J. H. McClellan, T. W. Parks, and L. R. Rabiner, "A computer program for designing optimum FIR linear phase digital filters," IEEE Transactions on Audio and Electroacoustics, vol. 21, no. 6, pp. 506-526, 1973.

Håkan Johansson was born in Kumla, Sweden, in 1969. He received the Master of Science degree in computer science and the $\mathrm{Li}$ centiate, Doctoral, and Docent degrees in electronics systems from Linköping University, Sweden, in 1995, 1997, 1998, and 2001, respectively. During 1998 and 1999 he held a post doctoral position at Signal Processing Laboratory, Tampere University of Technology, Finland. He is currently a Professor in

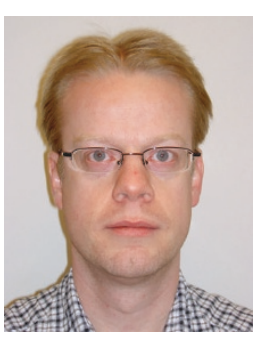
electronics systems at the Department of Electrical Engineering of Linköping University. His research interests include theory, design, and implementation of signal processing systems. He is the author or coauthor of four textbooks and more than 100 international journal and conference papers. He has served/serves as an Associate Editor for the IEEE Trans. on Circuits and Systems-II (2000-2001), IEEE Signal Processing Letters (2004-2007), and IEEE Trans. Signal Processing (2006-2008), and he is a Member of the IEEE Int. Symp. Circuits. Syst. DSP track committee.

Per Löwenborg was born in Oskarshamn, Sweden, in 1974. He received the Master of Science degree in applied physics and electrical engineering and the Licentiate, and Doctoral degrees in electronics systems from Linköping University, Sweden, in 1998, 2001, and 2002, respectively. His research interests are within the field of theory, design, and implementation of analog and digital signal processing electronics. He

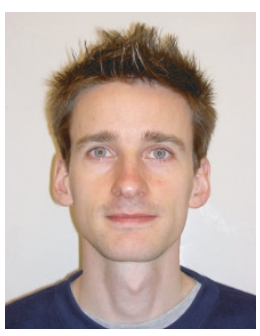
is the author or coauthor of one book and more than 50 international journal and conference papers. He was awarded the 1999 IEEE Midwest Symposium on Circuits and Systems best student paper award and the 2002 IEEE Nordic Signal Processing Symposium best paper award. He is a Member of the IEEE. 